\title{
Improved Surface Plasmon Resonance Sensing Sensitivity due to an Electrochemically Potential-Induced Gold Reconstruction
}

\author{
Baeck B. Choi ${ }^{1,2 *}$, Bethy Kim ${ }^{3}$, Yiqi Chen ${ }^{1}$, and Peng Jiang ${ }^{1 *}$ \\ ${ }^{1}$ Department of Chemical Engineering, University of Florida, Gainesville, FL 32611 USA \\ ${ }^{2}$ Energy and Environment Laboratory, Korea Electric Power Corp. Research Institute, Daejeon, 34056 Korea \\ ${ }^{3}$ Department of Chemistry, University of Florida, Gainesville, FL 32611 USA
}

\begin{abstract}
The progressively improved sensing sensitivity $\left(\Delta \lambda_{\mathrm{SPR}} / \Delta \mathrm{n}, \mathrm{nm} / \mathrm{RIU}\right)$ to detect the refractive index is observed on the SPR platform of an Au-covered epoxy gratings in an increase in potential cycling in a typical three-electrode cell. Here, a DVD$\mathrm{R}$ optical disc was used as a structure template to prepare an Au-covered epoxy gratings, and the newly formed reverse track pitch structure on the epoxy substrate was used as a working electrode directly in aqueous sulfuric acid solution. It is expected that Au reconstruction by potential cycling in sulfuric acid electrolyte increases the packing density of Au atoms in the grain boundary and improves the propagation of electromagnetic waves.
\end{abstract}

Keywords : Surface Plasmon Resonance, SPR, Au Reconstruction, Sensitivity

Received : 23 December 2020, Accepted : 20 January 2021

\section{Introduction}

Being highlighted label-free surface plasmon resonance (SPR) optical sensor uses modulation of the light output spectrum caused by locally modulated refractive index (localized surface plasmon resonance (LSPR)) [1-3]. For example, there is wavelength, angular distribution, intensity, phase, or polarization of the light output. Using a nanostructured grating coupler instead of using a large and complex prism-based platform (e.g., Kretschmann or Otto configurations) has the advantage of being simple and capable of scaling up. Nanostructured grating coupler is also being able to successively compensate the different wave-number between surface plasmon polaritons (SPPs) and external light. SPP is generated at an interface between metal and dielectric [4,5]. As a result, nanostructure-based grating couplers can successfully expand the practicality of plasmon sensors in a wide range of biosensors, photocatalytic,

*E-mail address: b.choi@kepco.co.kr, pjiang@che.ufl.edu DOI: https://doi.org/10.33961/jecst.2020.01767

This is an open-access article distributed under the terms of the Creative Commons Attribution Non-Commercial License (http://creativecommons.org/licenses/by-nc/4.0) Attribution Non-Commercial License (http://creativecommons.org/licenses/by-nc/4.0)
which permits unrestricted non-commercial use, distribution, and reproduction in any which permits unrestricted non-commercial use, distri
medium, provided the original work is properly cited. and heterogeneous catalytic researches [6-8].

In recent years, efforts have been made to develop nanostructure-based SPR platforms with high sensitivity $\left(\Delta \lambda_{\mathrm{SPR}} / \Delta \mathrm{n}, \mathrm{nm} / \mathrm{RIU}\right)$ and figure of merits (FOM, $\left.\left(\Delta \lambda_{\mathrm{SPR}} / \Delta \mathrm{n}\right) / \Delta \lambda, 1 / \mathrm{RIU}\right)$ in particular, in terms of easy scalability, feasible implementation, and low cost [4]. Due to the narrow bandwidth of the wavelength consumed by the excitation of the surface plasmons, a high FOM with a narrow full width half maximum (FWHM, nm) in the optical spectrum allows the resonance to be observed more clearly through an effective and accurate sensing process. For SPR to expand its application to various environmental conditions, the physical and chemical resistance of each component of the SPR platform as well as the SPR detection parameters with high SPR sensing sensitivity is important. Various bottom-up and top-down approaches have been taken for this strategy, and our research group has previously reported a highly sensitive SPR detection platform based on the track pitch structure of commercial optical discs such as CD, DVD and Blu-ray [6,9]. By applying a commercial optical disc as an SPR platform, it is possible to easily produce a large, scalable area with a simple and economically beneficial preparation method.

The sum of the binding energies of the surface 
atoms is defined as the surface energy. Therefore, the hexagonal close-packed structure of (111) facet with the highest packing density has the lowest surface energy [10]. There are three major ways to lower the surface energy. The first is the relaxation where the position of the atom changes but the unit cell remains unchanged. The second is reconstruction in which the periodicity of the unit cell and the surface changes, and the third is the formation of a surface oxide layer by ad-atoms or ad-molecules. In particular, surface relaxation or reconstruction is usually observed through thermal heat-treatment (or annealing, firing) or electrochemical treatment [11]. Finally, it causes accumulative energy density at a surface by smoothing effect and redistribution by missing neighbor. Severe heat treatment can lead to structural deformation, so we led Au reconstruction through electrochemical potential cycling, not heat treatment. Anyway, Au reconstruction by potential cycling in sulfuric acid electrolyte solution is a well-known phenomenon through many reports [10,12-14]. In short, the sequential and repetitive adsorption and desorption of sulfate anion and oxygen species on the $\mathrm{Au}$ electrode surface results in the rearrangement of $\mathrm{Au}$ atoms. In this report, we state that how this could affect the properties of surface plasmon as the optical sensors.

\section{Experimental}

Reconstruction of Au Gratings. A detailed fabrication method of Au-covered epoxy gratings was stated previously. Cyclic voltammetry (CV) was applied to a typical three-electrode cell in an aqueous sulfuric acid electrolyte solution ( $50 \mathrm{mmol} /$ liter $\mathrm{H}_{2} \mathrm{SO}_{4}$ ). Using a potentiostat/galvanostat instrument (263A, Princeton Applied Research), the electrode of $0.05 \mathrm{~V}$ to $1.60 \mathrm{~V}$ (vs. normal hydrogen electrode, NHE) was applied at a rate of $100 \mathrm{mV} / \mathrm{s} .1 .0 \mathrm{M} \mathrm{KCl}$ of $\mathrm{Ag} / \mathrm{AgCl}$ ( $\mathrm{CH}$ instruments) and $\mathrm{Pt}$ mesh (Sigma-Aldrich) were equipped as a reference and counter electrode, respectively.

\subsection{Physical Characteriaztions}

Scanning electron microscopy (SEM) images were taken on JEOL SEM-6400 (Major Analytical \& Particle Analysis Instrumentation Center (MAIC), University of Florida).

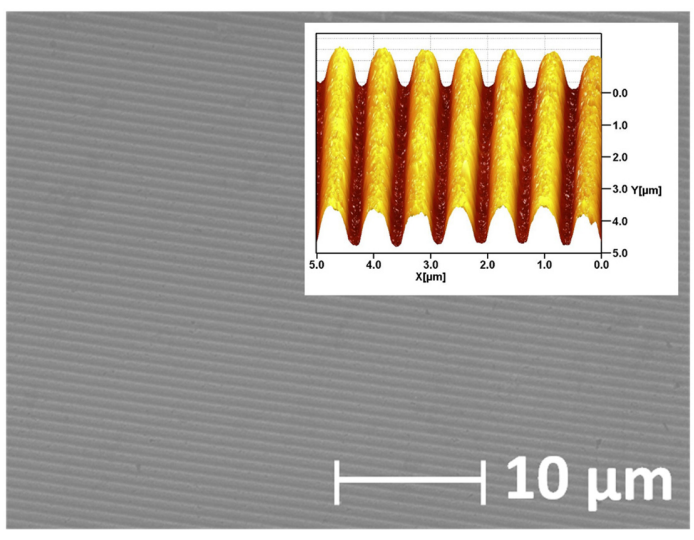

Fig. 1. SEM and AFM image of Au-covered epoxy gratings in which inverse track-pitch structure of DVD-R disc is formed.

\subsection{Normal-incidence Specular Optical Reflection Spectra and FDTD Simulation}

Incident normal reflection was measured by UVVis Spectrometer (HR4000, Ocean Optics) with halogen light source (DH-2000, Mikropack). SPR sensing tests were performed as a reference medium for standard refractive index in a home-made sandwich cell using de-ionized water (R.I., 1.332) and aqueous glycerol (Sigma Aldrich) solution (R.I., 1.343, 1.352, $1.363,1.372)$.

\section{Results and Discussion}

We have also previously observed a feasible manufacturing process for Au-covered epoxy gratings with inverse track-pitch (DVD-R) structure and those high SPR sensing sensitivity ( $~ 875 \mathrm{~nm} / \mathrm{RIU})$ and FOM $(\sim 110)[6,15]$. Au-covered epoxy grating was prepared by mixing epoxy resin (5 Minute Epoxy ${ }^{\mathrm{TM}}$, Devcon, bisphenol A diglycidyl ether (BADGE or DBEBA)) with a hardener (10-30\%, 2,4,6-tris[(dimethylamino)methyl]phenol) at room temperature firstly and well-mixed epoxy resin was poured into a $\mathrm{Au}$ layer ( $80 \mathrm{~nm}$ of thickness) surface which was sputtered onto the original polycarbonate (PC) substrate of DVD-R disc (Memorex ${ }^{\mathrm{TM}}$, 16X4.7GB, 120 $\mathrm{min}$ ). There is no adhesive thin metal layer (e.g., $\mathrm{Cr}$ or Ti) between Au layer and the PC board. Before injecting the mixed epoxy resin, removing a protective PC cover of DVD-R disc, washing organic dye with isopropyl alcohol (Sigma-Aldrich) and dissolv- 
ing $\mathrm{Al}$ recording layer out in nitric acid (98\%, SigmaAldrich) was done. After pouring the epoxy resin onto the sputtered Au layer of the PC board, it was placed in a dry oven at $100^{\circ} \mathrm{C}$ for 30 minutes. Next, it was peeled off the cured epoxy PC board. Cured epoxy was then peeled off from PC substrate. Through this simple cloning process of curing the epoxy resin, the sputtered Au layer of the PC board was continuously transferred to the epoxy board. Fig. 1, a scanning electron microscopy (SEM) image shows a very smooth Au surface on an epoxy substrate. In these easily prepared gratings, the inverse track-pitch structure of the DVD-R is successively formed with periodic narrow gorge geometry. Feasible delaminating cured epoxy from a PC substrate is enabled by strong adhesion between epoxy resin (surface tension, 43-47 dynes/cm) and the Au layer while here is a relatively weaker adhesion between $\mathrm{Au}$ layer and original PC substrate. During a tape test, no significant drop-out of Au layer was observed in the prepared Au-covered epoxy gratings and the $\mathrm{Au}$ layer of the PC substrate which is before Au layer transfer onto epoxy resin is easily delaminated. Compared to the Au layer of the original PC board, stronger physical properties can be obtained with a solid $\mathrm{Au}$ layer that is firmly attached and fixed to the epoxy substrate. Additionally, the FOM of SPR detection is dramatically improved from 52 to 110 by transferring the Au layer from PC to the epoxy substrate.

In addition, we try to induce electrochemically potential-induced Au reconstruction by applying potential cycling to an Au-covered epoxy gratings $[16,17]$. The applied electrochemical potential (V) can act as a driving force for metal nucleation as well as further possible decomposition mechanisms. The so-called "potential-induced reconstruction" for single crystal Au electrodes with low Miller index (e.g., (100), (110) and (111)) is well known for the increase and stabilization of the electron density among surface $\mathrm{Au}$ atoms. In this process, the (100) and (110) facets as well as the already dense (111) facets can be reconstructed by sequential adsorption of $\mathrm{SO}_{3}{ }^{-}$and hydroxyl radical, $\mathrm{OH}^{-}$like other oxygen species in an aqueous sulfuric acid solution [10,11]. It is already well known that continuous $\mathrm{Au}$ reconstruction is owned by thermal or flame annealing process. These annealing processes for $\mathrm{Au}$ reconstruction are typically operated at 450 to $550^{\circ} \mathrm{C}$ and Unfortunately,

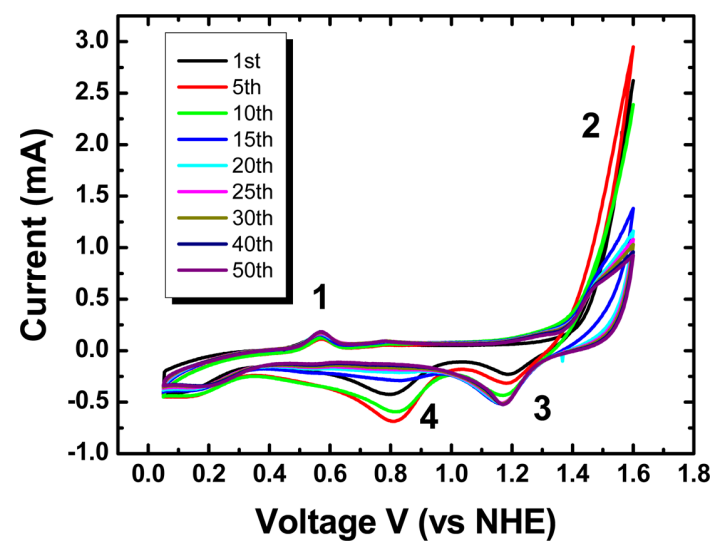

Fig. 2. Cyclic voltammograms of Au-covered epoxy gratings in nitrogen-purged aqueous $50 \quad \mathrm{mM} \quad \mathrm{H}_{2} \mathrm{SO}_{4}$ electrolyte solution with $100 \mathrm{mV} / \mathrm{s}$ of scan rate.

these temperature ranges can cause severe structural modifications to widely be used substrates for nanostructured lattice couplers, such as glass, silica, polystyrene (PS), and polymers. It has also been reported that metal layers annealed with a high temperature $\left(\sim 300^{\circ} \mathrm{C}\right)$ thermal annealing process showed dramatically grown Au islands and collapsed SPR signals (e.g., low intensity and wide SPR dips) [18-20]. In the case of electrochemical Au reconstruction, a reconstructed structure of the Au surface could be obtained without a dramatic change in the Au crystal structure even without introducing a thermal annealing process at high temperature.

$\mathrm{CV}$ was applied in an aqueous sulfuric acid electrolyte solution ( $50 \mathrm{mmol} / \mathrm{liter}$ ). Prior to using a Aucovered epoxy gratings as a working electrode directly, plastic-coated $\mathrm{Cu}$ wire was connected to the Au surface and additional insulation was done with epoxy resin to avoid possible contaminations. Before applying the potential cycling, inert nitrogen gas (UHP300, Airgas) was purged for more than 30 minutes to remove residual oxygen species in a liquid electrolyte solution. In Fig. 2, a distinct oxidation peak was observed during potential cycling at about $0.55 \mathrm{~V}$. This oxidation peak is well known for the adsorption of sulfuric acid groups $\left(\mathrm{SO}_{3}{ }^{-}\right)$on the $\mathrm{Au}$ electrode surface. Additional potential sweeps in the positive direction led to $\mathrm{Au}$ oxidation upon adsorption of oxygen species $\left(\mathrm{OH}^{-}, \mathrm{O}^{-}, \mathrm{O}_{2}^{-}\right)$. In the negative direction, the reduction of the adsorbed oxygen spe- 

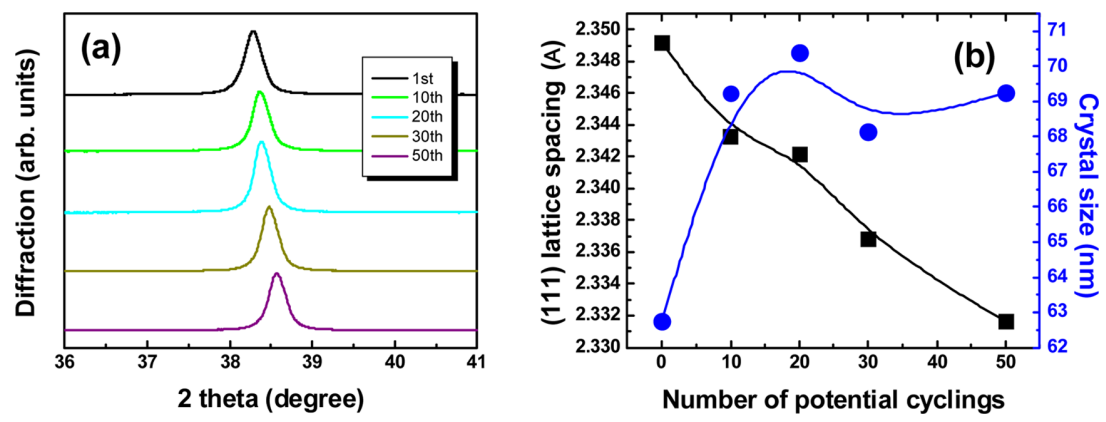

Fig. 3. (a) XRD patterns and (b) $\mathrm{Au}(111)$ lattice spacing and crystal size (nm) after potential cycling.

cies to water molecules $\left(\mathrm{H}_{2} \mathrm{O}\right)$ was observed at two distinct peak positions. The $\mathrm{SO}_{3}{ }^{-}$and $\mathrm{OH}^{-}$functional groups sequentially adsorbed on the Au surface restore and lift the Au monolayer from the electrode surface. Interestingly, as the number of applied potential cycles increases, the intensity of the oxygen reduction peak located at about $0.8 \mathrm{~V}$ gradually decreases and for $1.18 \mathrm{~V}$ it gradually increases. Similar results appear in the oxygen reduction reaction according to the physical properties of Pt nanoparticle electro-catalysts for the application of polymer electrolyte membrane fuel cells [21-23]. In particular, in the results of Brian E. Hayden et al, as the thickness of the Pt electrode prepared by physical vapor deposition increases, the peak potential of oxygen reduction in the cyclic voltammogram shifts to a high potential [23]. Also, in the carbon monoxide, $\mathrm{CO}$ stripping voltammogram, the thinner the Pt electrode, the higher the peak potential of CO stripping is. These results show that there is an early dramatic change in Au reconstruction by $\mathrm{CV}$ in sulfuric acid. These changes in the adsorption properties of nanoparticles are according to physical characteristics such as particle size. Instantly, the excess pressure acting on the internal bulk atoms of a particle from surface atoms present in a single particle is inversely proportional to the particle's radius.

Fig. 3 shows the changes in lattice spacing and crystal size according to XRD measurement of Aucovered epoxy gratings after potential cycling. It is clearly shown that the peak of Au (111) in XRD shifted to the higher high $2 \theta$ position, and the particle size calculated by the Debye-Scherrer equation also increased significantly from about 62.4 to $69.2 \mathrm{~nm}$ at the beginning of $\mathrm{CV}$. The clear changes in lattice spacing and crystal size in XRD measurements pre-
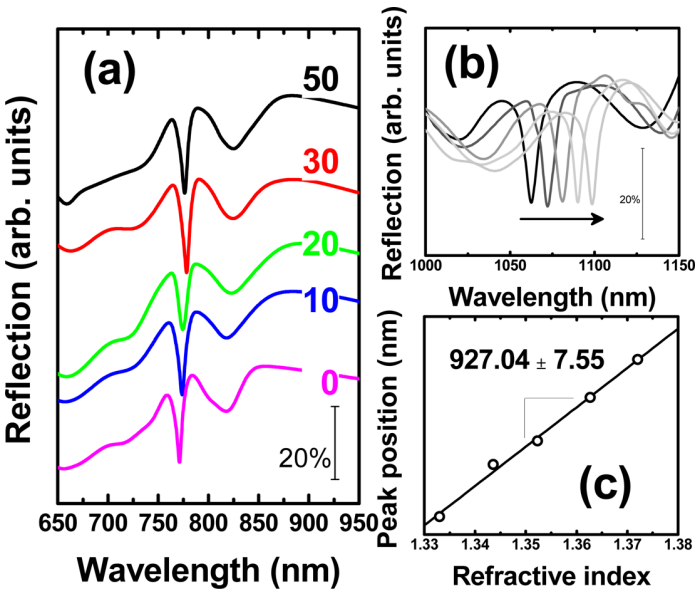

Fig. 4. (a) Incident normal reflection spectra in air after potential cycling. (b) SPR sensing sensitivity test of Aucoated epoxy gratings (50 potential cycling) with water and aqueous glycerol solutions and (c) its position vs. refractive index plot.

dicted changes in coherent delocalized electron oscillations between $\mathrm{Au}$ and dielectric interface due to an increase in packing density of gold atoms present in the grain boundary by electrochemical Au reconstruction by potential cycling.

The SPR detection sensitivity was monitored by measuring the normal incident specula optical reflection spectrum with a UV-Vis spectrometer (HR4000, Ocean Optics) and a halogen light source (DH-2000, Mikropack). During the detection test, the assembled home-made sandwich cell was equipped with optical fibers (Ocean Optics) and aqueous glycerol solutions $(0,9,16,24$ and $31 \%$ by weight $)$ to reference the refractive index. As we have already reported, a narrow SPR dip was observed in the air and aqueous 


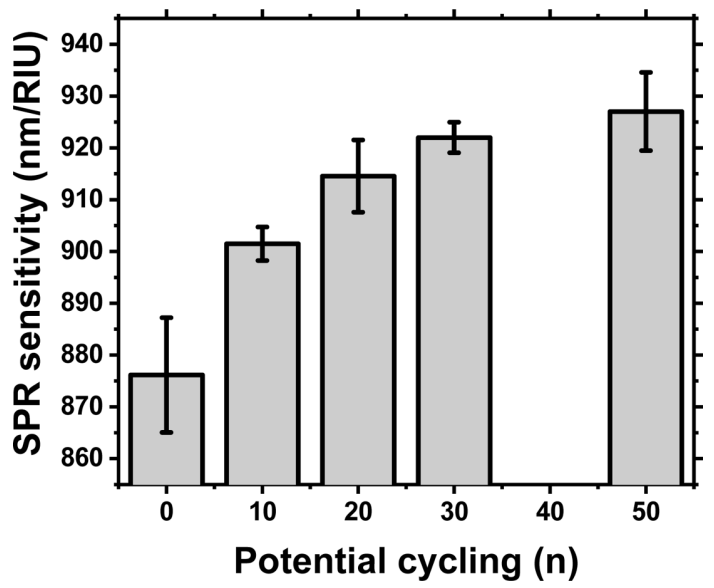

Fig. 5. SPR sensing sensitivity after potential cycling.

glycerol solution medium of an epoxy gratings covered with Au in Fig. 4(a, b). The SPR sensitivity obtained from the slope of the trend line between the refractive index in Fig. 4(c) and the SPR dip location $(\mathrm{nm})$ was summarized in Fig. 5 as the number of sequentially increased potential cycles. At least 3 samples were prepared for each potential cycle. Conditions and normal incidence specula optical spectra were measured at several locations on the surface of an epoxy grating covered with Au to obtain average SPR sensitivity and standard deviation. Prior to applying potential cycling to the Au-covered epoxy gratings, it exhibited an SPR detection sensitivity of $876.15 \pm 11.07 \mathrm{~nm} / \mathrm{RIU}$. After applying potential cycling, the SPR sensitivity gradually increases to $901.48 \pm 3.25$ (after 10 potential cycles), $914.56 \pm$ 6.96 ( 20 cycles), $921.99 \pm 2.95$ ( 30 cycles), and finally $927.04 \pm 7.55$ (50 cycles) in Fig. 5.

The representative sensing performance indicators of the surface plasmon sensor are sensitivity and figure of merit (FOM). The sensitivity, which is the sensitivity to the refractive index unit (RIU), represents the change in the position of the SPR dip per refractive index change [24-27]. FOM is a value obtained by dividing the sensitivity value by the FWHM of SPR dip, which is an index to evaluate the effectiveness and accuracy of the surface plasmon sensor [26,28-30]. The sensitivity of the plasmon sensor increases when the particle size constituting the metal or dielectric component of the plasmon sensor is large [9]. If the periodic pattern of the platform of surface plasmon decreases the aspect ratio through structural deformation, the sensitivity of the plasmon sensor does not decrease. Instead, the intensity of SPR dip decreases and FWHM increases, resulting in a decrease in FOM. This means that the accuracy of the plasmon sensor may be poor. However, in this result, it is not possible to see the change in SPR dip intensity or FWHM before and after potential cycling. If any impurities are present on the metal surface because of the cleanness issues, it is expected that the intensity or FWHM of the SPR dip will change because it affects the scattering or reflection of light on the surface of the plasmon substrate. However, this was also not confirmed in this result, and when the above results were combined, it can be concluded that the sensitivity of the plasmon sensor was improved without structural deformation when electrochemical $\mathrm{Au}$ reconstruction was selected instead of high temperature heat treatment.

\section{Conclusions}

The increased SPR sensing sensitivity in the Aucovered epoxy grating with reverse track-pitch structure is observed when applying potential cycling in aqueous sulfuric acid solution. The as-prepared grating shows $876.15 \pm 11.07 \mathrm{~nm} /$ RIU of sensing sensitivity, and finally increased to $927.04 \pm 7.55$ after 50 potential cycling. Observation of the XRD lattice spacing parameters reveal crystallographic modifications by electrochemically potential-induced $\mathrm{Au}$ reconstruction process. This is a way to improve sensitivity without affecting the accuracy of the plasmon sensor because structural deformation, which is a side effect of heat treatment at high temperatures, can be avoided.

\section{Acknowledgment}

This research was supported by the Korea Institute of Energy Technology Evaluation and Planning funded by the Korea government MOTIE (2019281010007A)

\section{References}

[1] J. Homola, Chem Rev. 2008, 108(2), 462-493.

[2] J. N. Anker, W. P. Hall, O. Lyandres, N. C. Shah, J. Zhao, R. P. Van Duyne, Nanosci Technol A Collect Rev from Nat Journals. 2009, 308-319. 
[3] K. A. Willets, R. P. Van Duyne, Annu Rev Phys Chem. 2007, 58, 267-297.

[4] H. C. Jeon, C. J. Heo, S. Y. Lee, S. G. Park, S. M. Yang, J Mater Chem. 2012, 22(11), 4603-4606.

[5] S. Y. Lee, H. C. Jeon, S. M. Yang, J Mater Chem. 2012, 22(13), 5900-5913.

[6] X. Dou, P. Y. Chung, P. Jiang, J. Dai, Appl Phys Lett. 2012, 100(4), 041116-041119.

[7] J. Yu, H. Tao, B. Cheng, ChemPhysChem. 2010, 11(8), 1617-1618.

[8] E. M. Larsson, C. Langhammer, I. Zorić, B. Kasemo, Science (80-). 2009, 326(5956), 1091-1094.

[9] B. Choi, X. Dou, Y. Fang, B. M. Phillips, P. Jiang, Phys Chem Chem Phys. 2016, 18(37), 26078-26087.

[10] T. Kondo, J. Morita, K. Hanaoka, S. Takakusagi, K. Tamura, M. Takahasi, J. N. I. Mizuki, K. Uosaki, J Phys Chem C. 2007, 111(35), 13197-13204.

[11] O. A. Hazzazi, G. A. Attard, P. B. Wells, F. J. VidalIglesias, M. Casadesus, J Electroanal Chem. 2009, 625(2), 123-130.

[12] J. Wang, A. J. Davenport, H. S. Isaacs, B. M. Ocko, Science (80-). 1991, 255(5050), 1416-1418.

[13] J. Wang, B. M. Ocko, A. J. Davenport, H. S. Issacs, Phys Rev B. 1992, 46(16), 10321-10338.

[14] T. Kondo, J. Zegenhagen, S. Takakusagi, K. Uosaki, SurfSci. 2015, 631, 96-104.

[15] B. Choi, Surface Plasmon Resonance Based on Optical Discs and Silica Microsphere Monolayer, University Of Florida 2015.

[16] J. Schneider, D. M. Kolb, Surf Sci. 1988, 193(3), 579592.
[17] D. M. Kolb, J. Schneider, Surf Sci. 1985, 162(1-3), 764775 .

[18] R. R. Abbas, T. H. Richardson, A. Hobson, A. Hassan, T. R. Abbas, Colloids Surfaces A Physicochem Eng Asp. 2014, 444, 95-103.

[19] C. Yan, Y. Chen, A. Jin, M. Wang, X. Kong, X. Zhang, Y. Ju, L. Han, Appl Surf Sci. 2011, 258(1), 377-381.

[20] V. Švorčík, O. Kvítek, O. Lyutakov, J. Siegel, Z. Kolská, Appl Phys A Mater Sci Process. 2011, 102(3), 747-751.

[21] M. Shao, A. Peles, K. Shoemaker, Nano Lett. 2011, 11(9), 3714-3719.

[22] K. J. J. Mayrhofer, B. B. Blizanac, M. Arenz, V. R. Stamenkovic, P. N. Ross, N. M. Markovic, J Phys Chem B. 2005, 109(30), 14433-14440.

[23] B. E. Hayden, D. Pletcher, J. P. Suchsland, L. J. Williams, Phys Chem Chem Phys. 2009, 11(40), 91419148.

[24] Y. Xia, N. J. Halas, MRS Bull. 2005, 30, 338-348.

[25] C. Noguez, J Phys Chem C. 2007, 111(10), 3606-3619.

[26] Y. Shen, J. Zhou, T. Liu, Y. Tao, R. Jiang, M. Liu, G. Xiao, J. Zhu, Z. K. Zhou, X. Wang, et al., Nat Commun. 2013, 4, 1-9.

[27] Y. Gao, Q. Gan, F. J. Bartoli, IEEE J Sel Top Quantum Electron. 2014, 20(3).

[28] Y. Xiong, J. Chen, B. Wiley, Y. Xia, Y. Yin, Z. Y. Li, Nano Lett. 2005, 5(7), 1237-1242.

[29] J. Henzie, M. H. Lee, T. W. Odom, Nat Nanotechnol. 2007, 2(9), 549-554.

[30] K. M. Mayer, J. H. Hafner, Chem Rev. 2011, 111(6), 3828-3857. 